\title{
PREreview of bioRxiv article "Ptr1 evolved convergently with RPS2 and Mr5 to mediate recognition of AvrRpt2 in diverse solanaceous species"
}

\author{
Matthew Helm ${ }^{1}$ and Morgan E. Carter ${ }^{2}$ \\ ${ }^{1}$ PREreview \\ ${ }^{2}$ Affiliation not available
}

April 28, 2020

Reviewers: Matthew Helm ${ }^{1}$ and Morgan E. Carter ${ }^{2}$

1. USDA-ARS, Crop Protection and Pest Control Research Unit, West Lafayette, IN

2. Plant Pathology and Plant-Microbe Biology Section, School of Integrative Plant Science, Cornell University, Ithaca, NY

\section{Summary}

This is a review of Mazo-Molina and Mainiero, et al. bioRxiv (doi: https://doi.org/10.1101/2020.03. 05.979484) posted on March 5, 2020. This study builds upon previous work that described the serendipitous discovery of a locus in the distantly related relative of tomato Solanum lycopersicoides, known as Pseudomonas tomato race 1 (Ptr1) (Mazo-Molina et al., (2019). Mazo-Molina and colleagues (2019) previously showed the Ptr1 locus confers resistance to several Pseudomonas syringae pv. tomato race 1 strains, but not the race 0 strain DC3000. Comparative genomic analysis of the effector repertoire between a race 1 strain of P. syringae pv. tomato, T1, and DC3000 identified AvrRpt2 as the cognate effector recognized by the Ptr1 locus. In Arabidopsis, AvrRpt2 is a cysteine protease that cleaves RIN4 and, upon cleavage, leads to activation of the Arabidopsis NLR protein RPS2.

In this paper, Mazo-Molina and Mainiero et al. describe the identification and functional characterization of the Ptr1 gene. A single recombinant among 585 F2 S. lycopersicoides individuals segregating for the Ptr1 locus was identified that narrowed the Ptr1 candidate to eight NLR genes. By employing currently available gene models for S. lycopersicoides and 3' RNA-seq data, Mazo-Molina and Mainiero et al. narrowed the list of Ptr 1 candidates to three genes; $A, B$, and $D$. Candidate $D$ was shown to be a pseudogene as it contained multiple mutations that disrupted the reading frame and was, therefore, not included in their functional analyses. Co-expression of candidate A with either AvrRpt2 or RipBN in N. glutinosa induced an HR-like cell death response. Candidate B, however, did not induce cell death by itself or when co-expressed with either AvrRpt2 or RipBN, and was thus not considered to be the Ptr1 gene. Taken together these experiments demonstrated that candidate A is the Ptr1 gene. Moreover, they show Ptr1 is conserved in many species within the Solanaceae family and that the Ptr1 ortholog from N. benthamiana and potato also mediate recognition of AvrRpt2 and RipBN. Lastly, phylogenetic analyses indicate that Ptr1, RPS2, and Mr5 (an NLR gene from apple whose protein product recognizes AvrRpt2) are not orthologous. The authors thus conclude the ability to recognize AvrRpt2 protease activity evolved convergently. 


\section{Comments}

Results section - LA4277 genome. In the first paragraph of the results section, the authors discuss NLRencoding genes in the S. lycopersicoides genome sequence as well as in LA4277-R. Later, it was mentioned that the LA4277 genome was assembled as part of this paper. We suggest clarifying at the first introduction of the LA4277 genome features that this genome was sequenced in this study, for readers unfamiliar with available genomes.

Results section - cell death assays. To test whether AvrRpt2 causes degradation of SlRin4 proteins resulting in activation of candidate A, the authors carried out cell death assays (Figure 2) by transiently co-expressing candidate A along with SlRin4-3 and AvrRpt2 or RipBN in N. glutinosa. However, in Figure $2 \mathrm{~A}$, they show transient co-expression of candidate A with either AvrRpt2 or RipBN is sufficient to induce an HR like cell death response, suggesting candidate A is guarding an endogenous RIN4-like protein in $N$. glutinosa. Did the authors try silencing the endogenous RIN4 protein in N. glutinosa and then co-express AvrRpt2/RipBN, candidate A, and SlRin4-3 to test whether SlRin4-3 has a role in AvrRpt2 recognition? We would also suggest performing an electrolyte leakage assay to quantify the cell death responses in Figure $2 \mathrm{C}$.

Results section-Figure 2C. In the experiment depicted in Figure 2C, what was the rationale behind including only SlRin4-3 and not the other additional Rin4 proteins?

Candidate B. Based on the immunoblot analysis in supplemental figure S2, candidate B protein expression is considerably weaker than that of candidate A protein expression (Figure 2D). Could it be a formal possibility that candidate B recognizes AvrRpt2 and RipBN, but because of the weak protein accumulation, there is no observable cell death in $N$. glutinosa?

Discussion section. As part of a very thorough discussion section, the second paragraph on page 10 covering RIN4 structure did not seem as relevant to the findings in this paper, as detailed analysis of Ptr1 detection of RIN4 cleavage was not experimentally investigated. 